\title{
Oscillation criteria for $p$-Laplacian delay dynamic equations with damping on time scales
}

\section{Zhenlai Han*, Yibing Sun and Meirong Xu}

\section{"Correspondence:} hanzhenlai@163.com

School of Mathematical Sciences, University of Jinan, Jinan, Shandong 250022, P.R. China

\begin{abstract}
In this paper, we establish some new oscillation criteria for $p$-Laplacian delay dynamic equations with damping

$$
\left(a(t) \Phi\left(x^{\Delta}(t)\right)\right)^{\Delta}+p(t) \Phi\left(x^{\Delta}(t)\right)+q(t) f(\Phi(x(\tau(t))))=0
$$

on a time scale $\mathbb{T}$, here $\Phi(s)=|s|^{\gamma-2} s, \gamma>1$ with $a, p$ and $q$ are positive real-valued rd-continuous functions defined on $\mathbb{T}$. By using the Riccati transformation technique and integral averaging technique, some new sufficient conditions, which ensure that every solution oscillates, are established. Our results in this paper not only extend and improve the known results, but also unify the results about oscillation criteria for $p$-Laplacian delay differential equations with damping and $p$-Laplacian delay difference equations with damping.

MSC: 34C10; 34K11; 39A21
\end{abstract}

Keywords: oscillation; second order; $p$-Laplacian delay dynamic equations; time scales

\section{Introduction}

In this paper, we are concerned with the oscillation criteria for the following $p$-Laplacian delay dynamic equations with damping:

$$
\left(a(t) \Phi\left(x^{\Delta}(t)\right)\right)^{\Delta}+p(t) \Phi\left(x^{\Delta}(t)\right)+q(t) f(\Phi(x(\tau(t))))=0, \quad t \in \mathbb{T}
$$

where $\Phi(s)=|s|^{\gamma-2} s, \gamma>1$. Throughout this paper and without further mention, we formulate the following hypotheses:

$\left(\mathrm{C}_{1}\right) \quad a, p, q: \mathbb{T} \rightarrow \mathbb{R}$ are positive rd-continuous functions such that $-p / a \in \mathcal{R}^{+}$;

$\left(C_{2}\right) \tau: \mathbb{T} \rightarrow \mathbb{T}, \tau(t) \leq t, \tau^{\Delta}(t)>0$ for all $t \in \mathbb{T}, \lim _{t \rightarrow \infty} \tau(t)=\infty$ and $\tilde{\mathbb{T}}=\tau(\mathbb{T}) \subset \mathbb{T}$ is a time scale;

$\left(C_{3}\right) f: \mathbb{R} \rightarrow \mathbb{R}$ is a continuous function such that for some positive constant $L, f(x) / x \geq L$ for all nonzero $x$.

The theory of time scales, which has recently received a lot of attention, was originally introduced by Stefan Hilger in his Ph.D. Thesis in 1988 in order to unify, extend and generalize continuous and discrete analysis (see Hilger [1]). A book on the subject of time scales

\section{第 Springer}

(c) 2013 Han et al.; licensee Springer. This is an Open Access article distributed under the terms of the Creative Commons Attribution License (http://creativecommons.org/licenses/by/2.0), which permits unrestricted use, distribution, and reproduction in any medium, provided the original work is properly cited. 
by Bohner and Peterson [2,3] summarizes and organizes much of the time scale calculus and many applications.

In recent years, there has been an increasing interest in obtaining sufficient conditions for the oscillation and nonoscillation of solutions of various equations on time scales; we refer the reader to the papers [4-18] and the references cited therein. For the oscillation results for second-order nonlinear dynamic equations with damping term, see, for example, [19-26]. To the best of our knowledge, it seems to have few oscillation results for the $p$-Laplacian dynamic equations with damping term; we refer the reader to the paper [27].

A time scale $\mathbb{T}$ is an arbitrary nonempty closed subset of real numbers $\mathbb{R}$, and the cases when this time scale is equal to the reals or to the integers represent the classical theories of differential and of difference equations. Not only does the new theory of the so-called dynamic equations unify the theories of differential equations and difference equations, but also it extends these classical cases to cases 'in between', for example, to the so-called $q$-difference equations when $\mathbb{T}=q^{\mathbb{N}_{0}}=\left\{q^{t}: t \in \mathbb{N}_{0}, q>1\right\}$ (which has important applications in quantum theory) and can be applied to different types of time scales like $\mathbb{T}=h \mathbb{N}$, $\mathbb{T}=\mathbb{N}^{2}$ and $\mathbb{T}=\mathbb{T}_{n}$ the space of the harmonic numbers.

Since we are interested in the oscillatory behavior of solutions near infinity, we make the assumption throughout this paper that the given time scale $\mathbb{T}$ is unbounded above. We assume that $t_{0} \in \mathbb{T}$ and it is convenient to assume that $t_{0}>0$. We define the time scale interval of the form $\left[t_{0}, \infty\right)_{\mathbb{T}}$ by $\left[t_{0}, \infty\right)_{\mathbb{T}}=\left[t_{0}, \infty\right) \cap \mathbb{T}$. We assume throughout that $\mathbb{T}$ has the topology that it inherits from the standard topology on the real numbers $\mathbb{R}$.

On any time scale, we define the forward and backward jump operators by

$$
\sigma(t)=\inf \{s \in \mathbb{T}: s>t\} \quad \text { and } \quad \rho(t)=\sup \{s \in \mathbb{T}: s<t\}
$$

where $\inf \emptyset=\sup \mathbb{T}$ and $\sup \emptyset=\inf \mathbb{T}$.

A point $t \in \mathbb{T}$ is said to be left-dense if $\rho(t)=t$, right-dense if $\sigma(t)=t$, left-scattered if $\rho(t)<t$ and right-scattered if $\sigma(t)>t$. A function $g: \mathbb{T} \rightarrow \mathbb{R}$ is said to be rd-continuous provided $g$ is continuous at right-dense points and at left-dense points in $\mathbb{T}$, left-hand limits exist and are finite. The set of all such rd-continuous functions is denoted by $C_{\mathrm{rd}}(\mathbb{T})$. The graininess function $\mu$ for a time scale $\mathbb{T}$ is defined by $\mu(t)=\sigma(t)-t$ and for any function $f: \mathbb{T} \rightarrow \mathbb{R}$, the notation $f^{\sigma}$ denotes $f \circ \sigma$.

By a solution of Eq. (1.1), we mean a nontrivial real-valued function $x$ satisfying Eq. (1.1) on $\left[t_{x}, \infty\right)_{\mathbb{T}}$. A solution $x$ of Eq. (1.1) is said to be oscillatory on $\left[t_{x}, \infty\right)_{\mathbb{T}}$ in case it is neither eventually positive nor eventually negative, otherwise it is nonoscillatory. Equation (1.1) is said to be oscillatory in case all its solutions are oscillatory. Our attention is restricted to those solutions of Eq. (1.1) which exist on some half-line $\left[t_{x}, \infty\right)_{\mathbb{T}}$ and satisfy $\sup \{|x(t)|$ : $t \geq T\}>0$ for all $T \geq t_{x}$.

Erbe et al. [22] investigated some oscillation criteria for the second-order nonlinear dynamic equations with damping

$$
\left(r(t)\left(x^{\Delta}(t)\right)^{\gamma}\right)^{\Delta}+p(t)\left(x^{\Delta \sigma}(t)\right)^{\gamma}+q(t) f(x(\tau(t)))=0, \quad t \in \mathbb{T},
$$

which extended and improved some known results. 
Saker et al. [23] considered the second-order dynamic equations with damping term of the form

$$
\left(r(t) x^{\Delta}(t)\right)^{\Delta}+p(t) x^{\Delta \sigma}(t)+q(t) f(x(\sigma(t)))=0, \quad t \in \mathbb{T},
$$

and by means of the Riccati transformation technique, established some sufficient conditions for oscillation of (1.3).

Chen et al. [26] studied the oscillation behavior of a class of second-order dynamic equations with damping

$$
\left(\left(x^{\Delta}(t)\right)^{\gamma}\right)^{\Delta}+p(t)\left(x^{\Delta}(t)\right)^{\gamma}+q(t) f\left(x^{\sigma}(t)\right)=0, \quad t \in \mathbb{T}
$$

where $\gamma$ is a quotient of odd positive integers, and established some sufficient conditions for oscillation of (1.4).

By using the Riccati transformation technique, inequalities and the Chain rule $(w \circ v)^{\Delta}=$ $\left(w^{\tilde{\Delta}} \circ v\right) v^{\Delta}$, where $\tilde{\Delta}$ is the delta derivative defined on $\tilde{\mathbb{T}}=v(\mathbb{T})$ and $v$ is strictly increasing, Zhang [27] studied the oscillatory behavior of all solutions of Eq. (1.1).

Note that the results in [27] are based on the condition $\tau(\mathbb{T})=\mathbb{T}$, which can be a restrictive condition and it is not easy to satisfy. For instance, when $\mathbb{T}=\{2,4,6, \ldots\}$ and letting $\tau(t)=t / 2$, then $\tau$ is a strictly increasing function, $\tau(t) \leq t$ and $\lim _{t \rightarrow \infty} \tau(t)=\infty$, but $\tau(\mathbb{T})=\{1,2,3, \ldots\} \neq \mathbb{T}$, so the condition $\tau(\mathbb{T})=\mathbb{T}$ in [27] does not hold and the results in [27] may not be true.

Hence, it would be interesting to study the oscillation behavior of Eq. (1.1) when $\tau(\mathbb{T})=\mathbb{T}$ does not hold.

We shall consider the two cases:

$$
\int_{t_{0}}^{\infty}\left[\frac{1}{a(t)} e_{-p / a}\left(t, t_{0}\right)\right]^{\frac{1}{\gamma-1}} \Delta t=\infty
$$

and

$$
\int_{t_{0}}^{\infty}\left[\frac{1}{a(t)} e_{-p / a}\left(t, t_{0}\right)\right]^{\frac{1}{\gamma-1}} \Delta t<\infty
$$

The paper is organized as follows. In Section 2, we present some lemmas which play important roles in the proofs of the main results. In Section 3, we intend to use the Riccati transformation technique, integral averaging technique and inequalities to obtain some sufficient conditions for oscillation of every solution of Eq. (1.1). In Section 4, we give an example in order to illustrate the main result.

\section{Some preliminary lemmas}

In order to prove our main results, we use the following formula:

$$
\left((x(t))^{\gamma}\right)^{\Delta}=\gamma \int_{0}^{1}\left[h x^{\sigma}(t)+(1-h) x(t)\right]^{\gamma-1} x^{\Delta}(t) \mathrm{d} h,
$$

where $x$ is delta differentiable and eventually positive or eventually negative, which is a simple consequence of Keller's chain rule (see [2, Theorem 1.90]). 
It is convenient to make the following notations:

$$
\begin{aligned}
& d_{+}(t):=\max \{0, d(t)\}, \quad d_{-}(t):=\max \{0,-d(t)\}, \\
& R(t)=a^{\frac{1}{\gamma-1}}(t) \int_{t_{1}}^{t}\left(\frac{1}{a(s)}\right)^{\frac{1}{\gamma-1}} \Delta s, \quad \alpha(t)=\frac{R(t)}{R(t)+\mu(t)}, \quad \theta(t)=\int_{t}^{\infty}\left(\frac{1}{a(s)}\right)^{\frac{1}{\gamma-1}} \Delta s,
\end{aligned}
$$

where $t_{1}$ is sufficiently large with $t_{1} \geq t_{0}$.

Next, we begin with the following lemmas which will be used in the proof of our main results.

Lemma 2.1 ([2, Chapter 2]) If $g \in \mathcal{R}^{+}$, i.e., $g: \mathbb{T} \rightarrow \mathbb{R}$ is rd-continuous and such that $1+$ $\mu(t) g(t)>0$ for all $t \in\left[t_{0}, \infty\right)_{\mathbb{T}}$, then the initial value problem $y^{\Delta}=g(t) y, y\left(t_{0}\right)=y_{0} \in \mathbb{R}$ has a unique and positive solution on $\left[t_{0}, \infty\right)_{\mathbb{T}}$, denoted by $e_{g}\left(\cdot, t_{0}\right)$. This 'exponential function' satisfies the semigroup property $e_{g}(a, b) e_{g}(b, c)=e_{g}(a, c)$.

Lemma 2.2 ([13, Lemma 2.2]) Assume that $\tau: \mathbb{T} \rightarrow \mathbb{T}$ is strictly increasingand $\tilde{\mathbb{T}}:=\tau(\mathbb{T}) \subset$ $\mathbb{T}$ is a time scale, $\tau \circ \sigma=\sigma \circ \tau$. Let $x: \tilde{\mathbb{T}} \rightarrow \mathbb{R}$. If $\tau^{\Delta}(t)$ and $x^{\Delta}(\tau(t))$ exist for $t \in \mathbb{T}^{\kappa}$, then $(x(\tau(t)))^{\Delta}$ exists, and

$$
(x(\tau(t)))^{\Delta}=x^{\Delta}(\tau(t)) \tau^{\Delta}(t) .
$$

Lemma 2.3 Assume that (1.5) holds. Furthermore, assume that $x$ is an eventually positive solution of (1.1). Then there exists $t_{1} \in\left[t_{0}, \infty\right)_{\mathbb{T}}$ such that

$$
\begin{aligned}
& x^{\Delta}(t)>0, \quad\left(a(t) \Phi\left(x^{\Delta}(t)\right)\right)^{\Delta}<0, \quad x(t)>R(t) x^{\Delta}(t), \\
& \frac{x(t)}{x^{\sigma}(t)}>\alpha(t), \quad t \in\left[t_{0}, \infty\right)_{\mathbb{T}} .
\end{aligned}
$$

Proof Let $x$ be an eventually positive solution of (1.1). Then there exists $t_{1} \in\left[t_{0}, \infty\right)_{\mathbb{T}}$ such that $x(t)>0$ and $x(\tau(t))>0$ for all $t \in\left[t_{1}, \infty\right)_{\mathbb{T}}$. Proceeding as in the proof of Lemma 3.5 in [27], we have $x^{\Delta}(t)>0,\left(a(t) \Phi\left(x^{\Delta}(t)\right)\right)^{\Delta}<0, t \in\left[t_{1}, \infty\right)_{\mathbb{T}}$, which yields that

$$
x(t)>x(t)-x\left(t_{1}\right)=\int_{t_{1}}^{t}\left(\frac{a(s)\left(x^{\Delta}(s)\right)^{\gamma-1}}{a(s)}\right)^{\frac{1}{\gamma-1}} \Delta s \geq\left(a(t)\left(x^{\Delta}(t)\right)^{\gamma-1}\right)^{\frac{1}{\gamma-1}} \int_{t_{1}}^{t}\left(\frac{1}{a(s)}\right)^{\frac{1}{\gamma-1}} \Delta s,
$$

that is,

$$
x(t)>R(t) x^{\Delta}(t) .
$$

In view of (2.4) and $x^{\sigma}(t)=x(t)+\mu(t) x^{\Delta}(t)$, it is easy to get that

$$
\frac{x(t)}{x^{\sigma}(t)}>\alpha(t)
$$

The proof is complete. 
Lemma 2.4 ([28, Theorem 41]) If $X$ and $Y$ are nonnegative real numbers and $\lambda>1$, then

$$
X^{\lambda}-\lambda X Y^{\lambda-1}+(\lambda-1) Y^{\lambda} \geq 0,
$$

where the equality holds if and only if $X=Y$.

Lemma 2.5 ([2, Theorem 6.1]) Let $y, f \in C_{\mathrm{rd}}$ and $p \in \mathcal{R}^{+}$. Then

$$
y^{\Delta}(t) \leq p(t) y(t)+f(t) \quad \text { for all } t \in \mathbb{T}
$$

implies

$$
y(t) \leq y\left(t_{0}\right) e_{p}\left(t, t_{0}\right)+\int_{t_{0}}^{t} e_{p}(t, \sigma(\tau)) f(\tau) \Delta \tau \quad \text { for all } t \in \mathbb{T} .
$$

\section{Main results}

Now, we are in a position to state and prove the main results which guarantee that every solution of Eq. (1.1) oscillates.

Theorem 3.1 Assume that (1.5) holds and $\tau \circ \sigma=\sigma \circ \tau$. Furthermore, assume that there exists a positive function $\delta \in C_{\mathrm{rd}}^{1}\left(\left[t_{0}, \infty\right)_{\mathbb{T}}, \mathbb{R}\right)$ such that for sufficiently large $t_{1}$,

$$
\limsup _{t \rightarrow \infty} \int_{t_{1}}^{t}\left(L q(s)(\alpha(\tau(s)))^{\gamma-1}-\frac{a(\tau(s))\left|\frac{\delta^{\Delta}(s)}{\delta(s)}-\frac{p(s)}{a(s)}\right|^{\gamma}}{\gamma^{\gamma}\left(\tau^{\Delta}(s)\right)^{\gamma-1}}\right) \delta(s) \Delta s=\infty
$$

Then Eq. (1.1) is oscillatory on $\left[t_{0}, \infty\right)_{\mathbb{T}}$.

Proof Suppose to the contrary that Eq. (1.1) has a nonoscillatory solution $x$ on $\left[t_{0}, \infty\right)_{\mathbb{T}}$. Without loss of generality, we may assume that there exists $t_{1} \in\left[t_{0}, \infty\right)_{\mathbb{T}}$ such that $x(t)>0$, $x(\tau(t))>0$ for all $t \in\left[t_{1}, \infty\right)_{\mathbb{T}}$. We shall consider only this case, since the case when $x$ is eventually negative is similar. From (1.1), (2.3) and $\left(C_{3}\right)$, we have

$$
\left(a(t)\left(x^{\Delta}(t)\right)^{\gamma-1}\right)^{\Delta}+p(t)\left(x^{\Delta}(t)\right)^{\gamma-1}+L q(t)(x(\tau(t)))^{\gamma-1} \leq 0 .
$$

Define the function $\omega$ by

$$
\omega(t)=\delta(t) \frac{a(t)\left(x^{\Delta}(t)\right)^{\gamma-1}}{(x(\tau(t)))^{\gamma-1}}, \quad t \in\left[t_{1}, \infty\right)_{\mathbb{T}} .
$$

Then $\omega(t)>0$. Using the product rule and the quotient rule, we get

$$
\begin{aligned}
\omega^{\Delta}(t)= & \delta^{\Delta}(t)\left(\frac{a(t)\left(x^{\Delta}(t)\right)^{\gamma-1}}{(x(\tau(t)))^{\gamma-1}}\right)^{\sigma}+\delta(t)\left(\frac{a(t)\left(x^{\Delta}(t)\right)^{\gamma-1}}{(x(\tau(t)))^{\gamma-1}}\right)^{\Delta} \\
= & \delta^{\Delta}(t) \frac{a^{\sigma}(t)\left(x^{\Delta \sigma}(t)\right)^{\gamma-1}}{\left(x\left(\tau^{\sigma}(t)\right)\right)^{\gamma-1}}+\delta(t) \frac{\left(a(t)\left(x^{\Delta}(t)\right)^{\gamma-1}\right)^{\Delta}}{\left(x\left(\tau^{\sigma}(t)\right)\right)^{\gamma-1}} \\
& -\delta(t) \frac{a(t)\left(x^{\Delta}(t)\right)^{\gamma-1}\left((x(\tau(t)))^{\gamma-1}\right)^{\Delta}}{(x(\tau(t)))^{\gamma-1}\left(x\left(\tau^{\sigma}(t)\right)\right)^{\gamma-1}}
\end{aligned}
$$


where $x^{\Delta \sigma}(t)$ is shorthand for $\left(x^{\Delta}(t)\right)^{\sigma}$. Using Lemma 2.3 and $\left(C_{2}\right)$, it follows that

$$
\frac{1}{x(\tau(t))} \geq \frac{1}{x\left(\tau^{\sigma}(t)\right)}, \quad a(t)\left(x^{\Delta}(t)\right)^{\gamma-1} \geq a^{\sigma}(t)\left(x^{\Delta \sigma}(t)\right)^{\gamma-1}
$$

and

$$
x^{\Delta}(\tau(t)) \geq \frac{\left(a^{\sigma}(t)\right)^{\frac{1}{\gamma-1}}}{(a(\tau(t)))^{\frac{1}{\gamma-1}}} x^{\Delta \sigma}(t), \quad t \in\left[t_{1}, \infty\right)_{\mathbb{T}} .
$$

From (3.2), (3.3) and (3.4), we obtain

$$
\begin{aligned}
\omega^{\Delta}(t) \leq & \frac{\delta^{\Delta}(t)}{\delta^{\sigma}(t)} \omega^{\sigma}(t)-\delta(t) p(t) \frac{\left(x^{\Delta}(t)\right)^{\gamma-1}}{\left(x\left(\tau^{\sigma}(t)\right)\right)^{\gamma-1}} \\
& -L \delta(t) q(t) \frac{(x(\tau(t)))^{\gamma-1}}{\left(x\left(\tau^{\sigma}(t)\right)\right)^{\gamma-1}}-\delta(t) \frac{a(t)\left(x^{\Delta}(t)\right)^{\gamma-1}\left((x(\tau(t)))^{\gamma-1}\right)^{\Delta}}{(x(\tau(t)))^{\gamma-1}\left(x\left(\tau^{\sigma}(t)\right)\right)^{\gamma-1}} .
\end{aligned}
$$

It follows from (2.5), (3.5) and (3.7) that

$$
\begin{aligned}
\omega^{\Delta}(t) \leq & \frac{\delta^{\Delta}(t)}{\delta^{\sigma}(t)} \omega^{\sigma}(t)-\frac{\delta(t) p(t)}{\delta^{\sigma}(t) a(t)} \omega^{\sigma}(t) \\
& -L \delta(t) q(t)(\alpha(\tau(t)))^{\gamma-1}-\delta(t) \frac{a(t)\left(x^{\Delta}(t)\right)^{\gamma-1}\left((x(\tau(t)))^{\gamma-1}\right)^{\Delta}}{(x(\tau(t)))^{\gamma-1}\left(x\left(\tau^{\sigma}(t)\right)\right)^{\gamma-1}} .
\end{aligned}
$$

If $\gamma>2$, from Lemmas 2.2, 2.3 and (2.1), we have

$$
\begin{aligned}
\left((x(\tau(t)))^{\gamma-1}\right)^{\Delta} & =(\gamma-1) \int_{0}^{1}\left[h x\left(\tau^{\sigma}(t)\right)+(1-h) x(\tau(t))\right]^{\gamma-2}(x(\tau(t)))^{\Delta} \mathrm{d} h \\
& \geq(\gamma-1)(x(\tau(t)))^{\gamma-2} x^{\Delta}(\tau(t)) \tau^{\Delta}(t) .
\end{aligned}
$$

From the above inequality and (3.5), we get

$$
\frac{\left((x(\tau(t)))^{\gamma-1}\right)^{\Delta}}{(x(\tau(t)))^{\gamma-1}} \geq \frac{(\gamma-1) x^{\Delta}(\tau(t)) \tau^{\Delta}(t)}{x\left(\tau^{\sigma}(t)\right)}, \quad t \in\left[t_{1}, \infty\right)_{\mathbb{T}} .
$$

Hence, from (3.6), (3.8) and (3.9), we obtain

$$
\begin{aligned}
\omega^{\Delta}(t) \leq & -L q(t) \delta(t)(\alpha(\tau(t)))^{\gamma-1}+\frac{\left|a(t) \delta^{\Delta}(t)-p(t) \delta(t)\right|}{a(t) \delta^{\sigma}(t)} \omega^{\sigma}(t) \\
& -\frac{(\gamma-1) \delta(t) a^{\sigma}(t)\left(x^{\Delta \sigma}(t)\right)^{\gamma-1} x^{\Delta}(\tau(t)) \tau^{\Delta}(t)}{\left(x\left(\tau^{\sigma}(t)\right)\right)^{\gamma}} \\
\leq & -L q(t) \delta(t)(\alpha(\tau(t)))^{\gamma-1}+\frac{\left|a(t) \delta^{\Delta}(t)-p(t) \delta(t)\right|}{a(t) \delta^{\sigma}(t)} \omega^{\sigma}(t) \\
& -\frac{(\gamma-1) \delta(t)\left(a^{\sigma}(t)\right)^{\frac{\gamma}{\gamma-1}}\left(x^{\Delta \sigma}(t)\right)^{\gamma} \tau^{\Delta}(t)}{(a(\tau(t))))^{\frac{1}{\gamma-1}}\left(x\left(\tau^{\sigma}(t)\right)\right)^{\gamma}} \\
\leq & -L q(t) \delta(t)(\alpha(\tau(t)))^{\gamma-1}+\frac{\left|a(t) \delta^{\Delta}(t)-p(t) \delta(t)\right|}{a(t) \delta^{\sigma}(t)} \omega^{\sigma}(t) \\
& -\frac{(\gamma-1) \delta(t) \tau^{\Delta}(t)}{(a(\tau(t))) \frac{1}{\gamma-1}\left(\delta^{\sigma}(t)\right)^{\frac{\gamma}{\gamma-1}}}\left(\omega^{\sigma}(t)\right)^{\frac{\gamma}{\gamma-1}} .
\end{aligned}
$$


If $1<\gamma \leq 2$, from Lemmas 2.2, 2.3 and (2.1), we have

$$
\left((x(\tau(t)))^{\gamma-1}\right)^{\Delta} \geq(\gamma-1)\left(x\left(\tau^{\sigma}(t)\right)\right)^{\gamma-2} x^{\Delta}(\tau(t)) \tau^{\Delta}(t) .
$$

Similarly to the proof of the previous case, we still get (3.10). Therefore, when $\gamma>1$, we obtain

$$
\begin{aligned}
\omega^{\Delta}(t) \leq & -L q(t) \delta(t)(\alpha(\tau(t)))^{\gamma-1}+\frac{\left|a(t) \delta^{\Delta}(t)-p(t) \delta(t)\right|}{a(t) \delta^{\sigma}(t)} \omega^{\sigma}(t) \\
& -\frac{(\gamma-1) \delta(t) \tau^{\Delta}(t)}{(a(\tau(t)))^{\lambda-1}\left(\delta^{\sigma}(t)\right)^{\lambda}}\left(\omega^{\sigma}(t)\right)^{\lambda}
\end{aligned}
$$

where $\lambda=\gamma /(\gamma-1)$. Define $X$ and $Y$ by

$$
X=\left(\frac{(\gamma-1) \delta(t) \tau^{\Delta}(t)}{(a(\tau(t)))^{\lambda-1}\left(\delta^{\sigma}(t)\right)^{\lambda}}\right)^{\frac{1}{\lambda}} \omega^{\sigma}(t)
$$

and

$$
Y=\left(\frac{\left|a(t) \delta^{\Delta}(t)-p(t) \delta(t)\right|}{\lambda a(t) \delta^{\sigma}(t)}\left(\frac{(\gamma-1) \delta(t) \tau^{\Delta}(t)}{(a(\tau(t)))^{\lambda-1}\left(\delta^{\sigma}(t)\right)^{\lambda}}\right)^{-\frac{1}{\lambda}}\right)^{\frac{1}{\lambda-1}} .
$$

Hence by (2.6), it follows that

$$
\begin{aligned}
& \frac{\left|a(t) \delta^{\Delta}(t)-p(t) \delta(t)\right|}{a(t) \delta^{\sigma}(t)} \omega^{\sigma}(t)-\frac{(\gamma-1) \delta(t) \tau^{\Delta}(t)}{(a(\tau(t)))^{\lambda-1}\left(\delta^{\sigma}(t)\right)^{\lambda}}\left(\omega^{\sigma}(t)\right)^{\lambda} \\
& \leq \frac{1}{\gamma^{\gamma}}\left|\frac{a(t) \delta^{\Delta}(t)-p(t) \delta(t)}{a(t) \delta^{\sigma}(t)}\right|^{\frac{\lambda}{\lambda-1}}\left(\frac{\delta(t) \tau^{\Delta}(t)}{(a(\tau(t)))^{\lambda-1}\left(\delta^{\sigma}(t)\right)^{\lambda}}\right)^{-\frac{1}{\lambda-1}} \\
& =\frac{a(\tau(t))}{\gamma^{\gamma}\left(\delta(t) \tau^{\Delta}(t)\right)^{\gamma-1}}\left|\delta^{\Delta}(t)-\delta(t) \frac{p(t)}{a(t)}\right|^{\gamma} .
\end{aligned}
$$

In view of (3.11) and (3.12), we have

$$
\omega^{\Delta}(t) \leq-L q(t) \delta(t)(\alpha(\tau(t)))^{\gamma-1}+\frac{a(\tau(t))}{\gamma^{\gamma}\left(\delta(t) \tau^{\Delta}(t)\right)^{\gamma-1}}\left|\delta^{\Delta}(t)-\delta(t) \frac{p(t)}{a(t)}\right|^{\gamma} .
$$

Integrating (3.13) from $t_{1}$ to $t$, we get

$$
\begin{aligned}
-\omega\left(t_{1}\right) & \leq \omega(t)-\omega\left(t_{1}\right) \\
& \leq-\int_{t_{1}}^{t}\left[L q(s)(\alpha(\tau(s)))^{\gamma-1}-\frac{\left.a(\tau(s)) \mid \frac{\delta^{\Delta}(s)}{\delta(s)}-\frac{p(s)}{a(s)}\right)^{\gamma}}{\gamma^{\gamma}\left(\tau^{\Delta}(s)\right)^{\gamma-1}}\right] \delta(s) \Delta s,
\end{aligned}
$$

which leads to a contradiction with (3.1). This completes the proof.

Remark 3.1 Note that in the special case when $\mathbb{T}=\mathbb{R}$, then $\sigma(t)=t, \mu(t)=0$, and $\alpha(t)=1$, then (3.1) becomes the condition (4.1) in [27], so Theorem 4.1 in [27] is a special case of our results. 
Remark 3.2 From Theorem 3.1, we can obtain different conditions for oscillation of all solutions of (1.1) with different choices of $\delta$.

Taking $\delta(t)=1$ and $\delta(t)=t$ in Theorem 3.1 respectively, we have the following two results.

Corollary 3.1 Assume that (1.5) holds and $\tau \circ \sigma=\sigma \circ \tau$. If for sufficiently large $t_{1}$,

$$
\limsup _{t \rightarrow \infty} \int_{t_{1}}^{t}\left(L q(s)(\alpha(\tau(s)))^{\gamma-1}-\frac{a(\tau(s)) p^{\gamma}(s)}{(\gamma a(s))^{\gamma}\left(\tau^{\Delta}(s)\right)^{\gamma-1}}\right) \Delta s=\infty
$$

then Eq. (1.1) is oscillatory on $\left[t_{0}, \infty\right)_{\mathbb{T}}$.

Corollary 3.2 Assume that (1.5) holds and $\tau \circ \sigma=\sigma \circ \tau$. If for sufficiently large $t_{1}$,

$$
\limsup _{t \rightarrow \infty} \int_{t_{1}}^{t} s\left(L q(s)(\alpha(\tau(s)))^{\gamma-1}-\frac{a(\tau(s))\left|\frac{1}{s}-\frac{p(s)}{a(s)}\right|^{\gamma}}{\gamma^{\gamma}\left(\tau^{\Delta}(s)\right)^{\gamma-1}}\right) \Delta s=\infty
$$

then Eq. (1.1) is oscillatory on $\left[t_{0}, \infty\right)_{\mathbb{T}}$.

Theorem 3.2 Assume that (1.5) holds and $\tau \circ \sigma=\sigma \circ \tau$. Furthermore, assume that there exist $m \geq 1$ and a positive function $\delta \in C_{\mathrm{rd}}^{1}\left(\left[t_{0}, \infty\right)_{\mathbb{T}}, \mathbb{R}\right)$ such that for sufficiently large $t_{1}$,

$$
\limsup _{t \rightarrow \infty} \frac{1}{t^{m}} \int_{t_{1}}^{t}(t-s)^{m}\left(L q(s)(\alpha(\tau(s)))^{\gamma-1}-\frac{a(\tau(s))\left|\frac{\delta^{\Delta}(s)}{\delta(s)}-\frac{p(s)}{a(s)}\right|^{\gamma}}{\gamma^{\gamma}\left(\tau^{\Delta}(s)\right)^{\gamma-1}}\right) \delta(s) \Delta s=\infty .
$$

Then Eq. (1.1) is oscillatory on $\left[t_{0}, \infty\right)_{\mathbb{T}}$.

Proof Suppose to the contrary that Eq. (1.1) has a nonoscillatory solution $x$ on $\left[t_{0}, \infty\right)_{\mathbb{T}}$. Without loss of generality, we may assume that there exists $t_{1} \in\left[t_{0}, \infty\right)_{\mathbb{T}}$ such that $x(t)>0$, $x(\tau(t))>0$ for all $t \in\left[t_{1}, \infty\right)_{\mathbb{T}}$. We shall consider only this case, since the case when $x$ is eventually negative is similar. We define the function $\omega$ by (3.3) again and proceeding as in the proof of Theorem 3.1, we have (3.13). Multiplying (3.13) by $(t-s)^{m}$ and integrating from $t_{1}$ to $t$, we get

$$
\begin{aligned}
& \int_{t_{1}}^{t}(t-s)^{m}\left(L q(s)(\alpha(\tau(s)))^{\gamma-1}-\frac{a(\tau(s))\left|\frac{\delta^{\Delta}(s)}{\delta(s)}-\frac{p(s)}{a(s)}\right|^{\gamma}}{\gamma^{\gamma}\left(\tau^{\Delta}(s)\right)^{\gamma-1}}\right) \delta(s) \Delta s \\
& \quad \leq-\int_{t_{1}}^{t}(t-s)^{m} \omega^{\Delta}(s) \Delta s .
\end{aligned}
$$

Using the integration by parts formula, we arrive at

$$
\int_{t_{1}}^{t}(t-s)^{m} \omega^{\Delta}(s) \Delta s=\left.(t-s)^{m} \omega(s)\right|_{t_{1}} ^{t}-\int_{t_{1}}^{t}\left((t-s)^{m}\right)^{\Delta_{s}} \omega^{\sigma}(s) \Delta s .
$$

From the proof of Theorem 2.9 in [17], we obtain

$$
\left((t-s)^{m}\right)^{\Delta_{s}} \leq-m(t-\sigma(s))^{m-1},
$$


where $t \geq \sigma(s), m \geq 1$. Combining (3.15) with (3.16) and (3.17), we have

$$
\begin{aligned}
& \int_{t_{1}}^{t}(t-s)^{m}\left(L q(s)(\alpha(\tau(s)))^{\gamma-1}-\frac{a(\tau(s))\left|\frac{\delta^{\Delta}(s)}{\delta(s)}-\frac{p(s)}{a(s)}\right|^{\gamma}}{\gamma^{\gamma}\left(\tau^{\Delta}(s)\right)^{\gamma-1}}\right) \delta(s) \Delta s \\
& \quad \leq\left(t-t_{1}\right)^{m} \omega\left(t_{1}\right)-m \int_{t_{1}}^{t}(t-\sigma(s))^{m-1} \omega^{\sigma}(s) \Delta s \leq\left(t-t_{1}\right)^{m} \omega\left(t_{1}\right)
\end{aligned}
$$

that is,

$$
\frac{1}{t^{m}} \int_{t_{1}}^{t}(t-s)^{m}\left(L q(s)(\alpha(\tau(s)))^{\gamma-1}-\frac{a(\tau(s))\left|\frac{\delta^{\Delta}(s)}{\delta(s)}-\frac{p(s)}{a(s)}\right|^{\gamma}}{\gamma^{\gamma}\left(\tau^{\Delta}(s)\right)^{\gamma-1}}\right) \delta(s) \Delta s \leq\left(\frac{t-t_{1}}{t}\right)^{m} \omega\left(t_{1}\right),
$$

which contradicts (3.14). This completes the proof.

Theorem 3.3 Assume that (1.5) holds and $\tau \circ \sigma=\sigma \circ \tau$. Furthermore, assume that there exist $m \geq 1$ and a positive function $\delta \in C_{\mathrm{rd}}^{1}\left(\left[t_{0}, \infty\right)_{\mathbb{T}}, \mathbb{R}\right)$ such that for sufficiently large $t_{1}$,

$$
\begin{aligned}
& \limsup _{t \rightarrow \infty} \frac{1}{t^{m}} \int_{t_{1}}^{t}\left[L(t-s)^{m} q(s) \delta(s)(\alpha(\tau(s)))^{\gamma-1} \delta(s)\right. \\
& \left.-\left(\frac{P(t, s) \delta^{\sigma}(s)}{\gamma}\right)^{\gamma} \frac{a(\tau(s))}{\left((t-s)^{m} \delta(s) \tau^{\Delta}(s)\right)^{\gamma-1}}\right] \Delta s=\infty,
\end{aligned}
$$

where

$$
P(t, s)=(t-s)^{m} \frac{\left|a(s) \delta^{\Delta}(s)-p(s) \delta(s)\right|}{a(s) \delta^{\sigma}(s)}-m(t-\sigma(s))^{m-1} .
$$

Then Eq. (1.1) is oscillatory on $\left[t_{0}, \infty\right)_{\mathbb{T}}$.

Proof Suppose to the contrary that Eq. (1.1) has a nonoscillatory solution $x$ on $\left[t_{0}, \infty\right)_{\mathbb{T}}$. Without loss of generality, we may assume that there exists $t_{1} \in\left[t_{0}, \infty\right)_{\mathbb{T}}$ such that $x(t)>0$, $x(\tau(t))>0$ for all $t \in\left[t_{1}, \infty\right)_{\mathbb{T}}$. We shall consider only this case, since the case when $x$ is eventually negative is similar. We define the function $\omega$ by (3.3) as before and proceed as in the proof of Theorem 3.1 to obtain (3.11). Then from (3.11) we have

$$
\begin{aligned}
L q(t) \delta(t)(\alpha(\tau(t)))^{\gamma-1} \leq & -\omega^{\Delta}(t)+\frac{\left|a(t) \delta^{\Delta}(t)-p(t) \delta(t)\right|}{a(t) \delta^{\sigma}(t)} \omega^{\sigma}(t) \\
& -\frac{(\gamma-1) \delta(t) \tau^{\Delta}(t)}{(a(\tau(t)))^{\lambda-1}\left(\delta^{\sigma}(t)\right)^{\lambda}}\left(\omega^{\sigma}(t)\right)^{\lambda} .
\end{aligned}
$$

Multiplying the above inequality by $(t-s)^{m}$ and integrating from $t_{1}$ to $t$, we get

$$
\begin{aligned}
& L \int_{t_{1}}^{t}(t-s)^{m} q(s) \delta(s)(\alpha(\tau(s)))^{\gamma-1} \Delta s \\
& \quad \leq-\int_{t_{1}}^{t}(t-s)^{m} \omega^{\Delta}(s) \Delta s+\int_{t_{1}}^{t}(t-s)^{m} \frac{\left|a(s) \delta^{\Delta}(s)-p(s) \delta(s)\right|}{a(s) \delta^{\sigma}(s)} \omega^{\sigma}(s) \Delta s \\
& \quad-\int_{t_{1}}^{t}(t-s)^{m} \frac{(\gamma-1) \delta(s) \tau^{\Delta}(s)}{(a(\tau(s)))^{\lambda-1}\left(\delta^{\sigma}(s)\right)^{\lambda}}\left(\omega^{\sigma}(s)\right)^{\lambda} \Delta s .
\end{aligned}
$$


Combining (3.19) with (3.16) and (3.17), we obtain

$$
\begin{aligned}
& L \int_{t_{1}}^{t}(t-s)^{m} q(s) \delta(s)(\alpha(\tau(s)))^{\gamma-1} \Delta s \\
& \leq\left(t-t_{1}\right)^{m} \omega\left(t_{1}\right)+\int_{t_{1}}^{t}(t-s)^{m} \frac{\left|a(s) \delta^{\Delta}(s)-p(s) \delta(s)\right|}{a(s) \delta^{\sigma}(s)} \omega^{\sigma}(s) \Delta s \\
& \quad-m \int_{t_{1}}^{t}(t-\sigma(s))^{m-1} \omega^{\sigma}(s) \Delta s \\
& \quad-\int_{t_{1}}^{t}(t-s)^{m} \frac{(\gamma-1) \delta(s) \tau^{\Delta}(s)}{(a(\tau(s)))^{\lambda-1}\left(\delta^{\sigma}(s)\right)^{\lambda}}\left(\omega^{\sigma}(s)\right)^{\lambda} \Delta s .
\end{aligned}
$$

Define $X$ and $Y$ by

$$
X=\left((t-s)^{m} \frac{(\gamma-1) \delta(s) \tau^{\Delta}(s)}{(a(\tau(s)))^{\lambda-1}\left(\delta^{\sigma}(s)\right)^{\lambda}}\right)^{\frac{1}{\lambda}} \omega^{\sigma}(s)
$$

and

$$
Y=\left(\frac{P(t, s)}{\lambda}\left((t-s)^{m} \frac{(\gamma-1) \delta(s) \tau^{\Delta}(s)}{(a(\tau(s)))^{\lambda-1}\left(\delta^{\sigma}(s)\right)^{\lambda}}\right)^{-\frac{1}{\lambda}}\right)^{\frac{1}{\lambda-1}}
$$

Hence by (2.6), it follows that

$$
\begin{gathered}
P(t, s) \omega^{\sigma}(s)-(t-s)^{m} \frac{(\gamma-1) \delta(s) \tau^{\Delta}(s)}{(a(\tau(s)))^{\lambda-1}\left(\delta^{\sigma}(s)\right)^{\lambda}}\left(\omega^{\sigma}(s)\right)^{\lambda} \\
\leq\left(\frac{P(t, s) \delta^{\sigma}(s)}{\gamma}\right)^{\gamma} \frac{a(\tau(s))}{\left((t-s)^{m} \delta(s) \tau^{\Delta}(s)\right)^{\gamma-1}} .
\end{gathered}
$$

From (3.20) and (3.21), we have

$$
\begin{aligned}
& L \int_{t_{1}}^{t}(t-s)^{m} q(s) \delta(s)(\alpha(\tau(s)))^{\gamma-1} \Delta s-\int_{t_{1}}^{t}\left(\frac{P(t, s) \delta^{\sigma}(s)}{\gamma}\right)^{\gamma} \frac{a(\tau(s))}{\left((t-s)^{m} \delta(s) \tau^{\Delta}(s)\right)^{\gamma-1}} \Delta s \\
& \quad \leq\left(t-t_{1}\right)^{m} \omega\left(t_{1}\right),
\end{aligned}
$$

that is,

$$
\begin{aligned}
& \frac{1}{t^{m}} \int_{t_{1}}^{t}\left(L(t-s)^{m} q(s) \delta(s)(\alpha(\tau(s)))^{\gamma-1}-\left(\frac{P(t, s) \delta^{\sigma}(s)}{\gamma}\right)^{\gamma} \frac{a(\tau(s))}{\left((t-s)^{m} \delta(s) \tau^{\Delta}(s)\right)^{\gamma-1}}\right) \Delta s \\
& \quad \leq\left(\frac{t-t_{1}}{t}\right)^{m} \omega\left(t_{1}\right) .
\end{aligned}
$$

This is a contradiction with (3.18). This completes the proof.

Theorem 3.4 Assume that (1.5) holds and $\tau \circ \sigma=\sigma \circ \tau$. Furthermore, assume that there exist functions $H, h \in C_{\mathrm{rd}}(\mathbb{D}, \mathbb{R})$, where $\mathbb{D} \equiv\left\{(t, s): t \geq s \geq t_{0}\right\}$ such that

$$
H(t, t)=0, \quad t \geq t_{0}, \quad H(t, s)>0, \quad t>s \geq t_{0}
$$


and $H$ has a nonpositive continuous $\Delta$-partial derivation $H^{\Delta_{s}}(t, s)$ with respect to the second variable and satisfies

$$
H^{\Delta_{s}}(t, s)+H(t, s) \frac{\left|a(s) \delta^{\Delta}(s)-p(s) \delta(s)\right|}{a(s) \delta^{\sigma}(s)}=-\frac{h(t, s)}{\delta^{\sigma}(s)}(H(t, s))^{\frac{\gamma-1}{\gamma}}
$$

and for sufficiently large $t_{1}$,

$$
\limsup _{t \rightarrow \infty} \frac{1}{H\left(t, t_{1}\right)} \int_{t_{1}}^{t}\left(L H(t, s) q(s) \delta(s)(\alpha(\tau(s)))^{\gamma-1}-\frac{\left(h_{-}(t, s)\right)^{\gamma} a(\tau(s))}{\gamma^{\gamma}\left(\delta(s) \tau^{\Delta}(s)\right)^{\gamma-1}}\right) \Delta s=\infty,
$$

where $\delta \in C_{\mathrm{rd}}^{1}\left(\left[t_{0}, \infty\right)_{\mathbb{T}}, \mathbb{R}\right)$ is a positive function. Then Eq. (1.1) is oscillatory on $\left[t_{0}, \infty\right)_{\mathbb{T}}$.

Proof Suppose to the contrary that Eq. (1.1) has a nonoscillatory solution $x$ on $\left[t_{0}, \infty\right)_{\mathbb{T}}$. Without loss of generality, we may assume that there exists $t_{1} \in\left[t_{0}, \infty\right)_{\mathbb{T}}$ such that $x(t)>0$, $x(\tau(t))>0$ for all $t \in\left[t_{1}, \infty\right)_{\mathbb{T}}$. We shall consider only this case, since the case when $x$ is eventually negative is similar. We define the function $\omega$ by (3.3) as before and proceed as in the proof of Theorem 3.1 to obtain (3.11). Multiplying (3.11) by $H(t, s)$ and integrating from $t_{1}$ to $t$, we get

$$
\begin{aligned}
\int_{t_{1}}^{t} H(t, s) \omega^{\Delta}(s) \Delta s \leq & -L \int_{t_{1}}^{t} H(t, s) q(s) \delta(s)(\alpha(\tau(s)))^{\gamma-1} \Delta s \\
& +\int_{t_{1}}^{t} H(t, s) \frac{\left|a(s) \delta^{\Delta}(s)-p(s) \delta(s)\right|}{a(s) \delta^{\sigma}(s)} \omega^{\sigma}(s) \Delta s \\
& -\int_{t_{1}}^{t} H(t, s) \frac{(\gamma-1) \delta(s) \tau^{\Delta}(s)}{(a(\tau(s)))^{\lambda-1}\left(\delta^{\sigma}(s)\right)^{\lambda}}\left(\omega^{\sigma}(s)\right)^{\lambda} \Delta s .
\end{aligned}
$$

Integrating the left side by parts and from (3.22), we obtain

$$
\begin{aligned}
\int_{t_{1}}^{t} H(t, s) \omega^{\Delta}(s) \Delta s & =\left.H(t, s) \omega(s)\right|_{t_{1}} ^{t}-\int_{t_{1}}^{t} H^{\Delta_{s}}(t, s) \omega^{\sigma}(s) \Delta s \\
& =-H\left(t, t_{1}\right) \omega\left(t_{1}\right)-\int_{t_{1}}^{t} H^{\Delta_{s}}(t, s) \omega^{\sigma}(s) \Delta s .
\end{aligned}
$$

Therefore,

$$
\begin{aligned}
& L \int_{t_{1}}^{t} H(t, s) q(s) \delta(s)(\alpha(\tau(s)))^{\gamma-1} \Delta s \\
& \leq H\left(t, t_{1}\right) \omega\left(t_{1}\right)+\int_{t_{1}}^{t} H^{\Delta_{s}}(t, s) \omega^{\sigma}(s) \Delta s \\
& \quad+\int_{t_{1}}^{t} H(t, s) \frac{\left|a(s) \delta^{\Delta}(s)-p(s) \delta(s)\right|}{a(s) \delta^{\sigma}(s)} \omega^{\sigma}(s) \Delta s \\
& \quad-\int_{t_{1}}^{t} H(t, s) \frac{(\gamma-1) \delta(s) \tau^{\Delta}(s)}{(a(\tau(s)))^{\lambda-1}\left(\delta^{\sigma}(s)\right)^{\lambda}}\left(\omega^{\sigma}(s)\right)^{\lambda} \Delta s \\
& \leq H\left(t, t_{1}\right) \omega\left(t_{1}\right) \\
&+\int_{t_{1}}^{t}\left[-\frac{h(t, s)}{\delta^{\sigma}(s)}(H(t, s))^{\frac{\gamma-1}{\gamma}} \omega^{\sigma}(s)-H(t, s) \frac{(\gamma-1) \delta(s) \tau^{\Delta}(s)}{(a(\tau(s)))^{\lambda-1}\left(\delta^{\sigma}(s)\right)^{\lambda}}\left(\omega^{\sigma}(s)\right)^{\lambda}\right] \Delta s
\end{aligned}
$$




$$
\begin{aligned}
\leq & H\left(t, t_{1}\right) \omega\left(t_{1}\right) \\
& +\int_{t_{1}}^{t}\left[\frac{h_{-}(t, s)}{\delta^{\sigma}(s)}(H(t, s))^{\frac{1}{\lambda}} \omega^{\sigma}(s)-H(t, s) \frac{(\gamma-1) \delta(s) \tau^{\Delta}(s)}{(a(\tau(s)))^{\lambda-1}\left(\delta^{\sigma}(s)\right)^{\lambda}}\left(\omega^{\sigma}(s)\right)^{\lambda}\right] \Delta s .
\end{aligned}
$$

Define $X$ and $Y$ by

$$
X=\left(H(t, s) \frac{(\gamma-1) \delta(s) \tau^{\Delta}(s)}{(a(\tau(s)))^{\lambda-1}\left(\delta^{\sigma}(s)\right)^{\lambda}}\right)^{\frac{1}{\lambda}} \omega^{\sigma}(s)
$$

and

$$
Y=\left(\frac{h_{-}(t, s)}{\lambda \delta^{\sigma}(s)}\left(\frac{(\gamma-1) \delta(s) \tau^{\Delta}(s)}{(a(\tau(s)))^{\lambda-1}\left(\delta^{\sigma}(s)\right)^{\lambda}}\right)^{-\frac{1}{\lambda}}\right)^{\frac{1}{\lambda-1}}
$$

Hence by (2.6), it follows that

$$
\begin{aligned}
& \frac{h_{-}(t, s)}{\delta^{\sigma}(s)}(H(t, s))^{\frac{1}{\lambda}} \omega^{\sigma}(s)-H(t, s) \frac{(\gamma-1) \delta(s) \tau^{\Delta}(s)}{(a(\tau(s)))^{\lambda-1}\left(\delta^{\sigma}(s)\right)^{\lambda}}\left(\omega^{\sigma}(s)\right)^{\lambda} \\
& \quad \leq \frac{\left(h_{-}(t, s)\right)^{\gamma} a(\tau(s))}{\gamma^{\gamma}\left(\delta(s) \tau^{\Delta}(s)\right)^{\gamma-1}} .
\end{aligned}
$$

From (3.25) and (3.26), we get

$$
L \int_{t_{1}}^{t} H(t, s) q(s) \delta(s)(\alpha(\tau(s)))^{\gamma-1} \Delta s-\int_{t_{1}}^{t} \frac{\left(h_{-}(t, s)\right)^{\gamma} a(\tau(s))}{\gamma^{\gamma}\left(\delta(s) \tau^{\Delta}(s)\right)^{\gamma-1}} \Delta s \leq H\left(t, t_{1}\right) \omega\left(t_{1}\right),
$$

that is,

$$
\frac{1}{H\left(t, t_{1}\right)} \int_{t_{1}}^{t}\left[L H(t, s) q(s) \delta(s)(\alpha(\tau(s)))^{\gamma-1}-\frac{\left(h_{-}(t, s)\right)^{\gamma} a(\tau(s))}{\gamma^{\gamma}\left(\delta(s) \tau^{\Delta}(s)\right)^{\gamma-1}}\right] \Delta s \leq \omega\left(t_{1}\right),
$$

which is a contradiction with (3.24). This completes the proof.

Theorem 3.5 Assume that (1.6) holds and $\tau \circ \sigma=\sigma \circ \tau$. Furthermore, assume that there exists a positive function $\delta \in C_{\mathrm{rd}}^{1}\left(\left[t_{0}, \infty\right)_{\mathbb{T}}, \mathbb{R}\right)$ such that for sufficiently large $t_{1},(3.1)$ or (3.14), (3.18) and (3.24) hold, where $m \geq 1, P, H$ and $h$ are defined as in Theorems 3.3 and 3.4. If there exists a positive function $\eta \in C_{\mathrm{rd}}^{1}\left(\left[t_{0}, \infty\right)_{\mathbb{T}}, \mathbb{R}\right), \eta^{\Delta}(t) \geq 0$ such that

$$
\int_{t_{1}}^{\infty}\left(\frac{1}{\eta(t) a(t)} \int_{t_{1}}^{t} e_{-p / a}(t, \sigma(s)) \eta(s) q(s) \theta^{\gamma-1}(s) \Delta s\right)^{\frac{1}{\gamma-1}} \Delta t=\infty
$$

then Eq. (1.1) is oscillatory on $\left[t_{0}, \infty\right)_{\mathbb{T}}$.

Proof Suppose to the contrary that Eq. (1.1) has a nonoscillatory solution $x$ on $\left[t_{0}, \infty\right)_{\mathbb{T}}$. Without loss of generality, we may assume that there exists $t_{1} \in\left[t_{0}, \infty\right)_{\mathbb{T}}$ such that $x(t)>0$, $x(\tau(t))>0$ for all $t \in\left[t_{1}, \infty\right)_{\mathbb{T}}$. We shall consider only this case, since the case when $x$ is eventually negative is similar. Proceeding as in the proof of Lemma 3.5 in [27], we have

$$
\frac{a(t)\left|x^{\Delta}(t)\right|^{\gamma-2} x^{\Delta}(t)}{e_{-p / a}\left(t, t_{0}\right)} \text { is decreasing. }
$$


By Lemma 2.1, we get $x^{\Delta}(t)$ is either eventually positive or eventually negative. Hence, there are two possible cases.

Case (I). $x^{\Delta}(t)>0, t \in\left[t_{1}, \infty\right)_{\mathbb{T}}$.

The proof when $x^{\Delta}(t)$ is eventually positive is similar to that of Theorems 3.1 or 3.2, 3.3 and 3.4, so we omit the details.

Case (II). $x^{\Delta}(t)<0, t \in\left[t_{1}, \infty\right)_{\mathbb{T}}$.

From (3.28) and Lemma 2.1, we have

$$
a(s)\left|x^{\Delta}(s)\right|^{\gamma-2} x^{\Delta}(s) \leq a(t)\left|x^{\Delta}(t)\right|^{\gamma-2} x^{\Delta}(t), \quad s \in[t, \infty)_{\mathbb{T}},
$$

that is,

$$
a(s)\left|x^{\Delta}(s)\right|^{\gamma-1} \geq a(t)\left|x^{\Delta}(t)\right|^{\gamma-1}
$$

Hence

$$
-x^{\Delta}(s) \geq\left(\frac{a(t)}{a(s)}\right)^{\frac{1}{\gamma-1}}\left(-x^{\Delta}(t)\right)
$$

Integrating (3.29) from $t$ to $\infty$, we get

$$
x(t) \geq a^{\frac{1}{\gamma-1}}(t)\left(-x^{\Delta}(t)\right) \int_{t}^{\infty}\left(\frac{1}{a(s)}\right)^{\frac{1}{\gamma-1}} \Delta s \geq b \theta(t), \quad t \in\left[t_{1}, \infty\right)_{\mathbb{T}},
$$

where $b=a^{1 /(\gamma-1)}\left(t_{1}\right)\left(-x^{\Delta}\left(t_{1}\right)\right)>0$. Using (3.29) and the above inequality in Eq. (1.1), we obtain

$$
\begin{aligned}
- & \left(a(t)\left|x^{\Delta}(t)\right|^{\gamma-2} x^{\Delta}(t)\right)^{\Delta} \\
& =p(t)\left|x^{\Delta}(t)\right|^{\gamma-2} x^{\Delta}(t)+q(t) f\left(|x(\tau(t))|^{\gamma-2} x(\tau(t))\right) \\
& \geq p(t)\left|x^{\Delta}(t)\right|^{\gamma-2} x^{\Delta}(t)+L q(t)(x(\tau(t)))^{\gamma-1} \\
& \geq p(t)\left|x^{\Delta}(t)\right|^{\gamma-2} x^{\Delta}(t)+L q(t) x^{\gamma-1}(t) \\
& \geq p(t)\left|x^{\Delta}(t)\right|^{\gamma-2} x^{\Delta}(t)+L b^{\gamma-1} q(t) \theta^{\gamma-1}(t), \quad t \in\left[t_{1}, \infty\right)_{\mathbb{T}} .
\end{aligned}
$$

Define the function $u$ by

$$
u(t)=\eta(t) a(t)\left|x^{\Delta}(t)\right|^{\gamma-2} x^{\Delta}(t)=-\eta(t) a(t)\left|x^{\Delta}(t)\right|^{\gamma-1}, \quad t \in\left[t_{1}, \infty\right)_{\mathbb{T}} .
$$

Then $u(t)<0$. Using the product rule and from (3.30), we find that

$$
\begin{aligned}
u^{\Delta}(t) & =-\eta^{\Delta}(t)\left(a(t)\left|x^{\Delta}(t)\right|^{\gamma-1}\right)^{\sigma}-\eta(t)\left(a(t)\left|x^{\Delta}(t)\right|^{\gamma-1}\right)^{\Delta} \\
& \leq-\eta(t) p(t)\left|x^{\Delta}(t)\right|^{\gamma-2} x^{\Delta}(t)-L b^{\gamma-1} \eta(t) q(t) \theta^{\gamma-1}(t) \\
& =-\frac{p(t)}{a(t)} u(t)-L b^{\gamma-1} \eta(t) q(t) \theta^{\gamma-1}(t) .
\end{aligned}
$$


Since $-p / a \in \mathcal{R}^{+}$, then from Lemma 2.5 , it follows

$$
\begin{aligned}
u(t) & \leq u\left(t_{1}\right) e_{-p / a}\left(t, t_{1}\right)-L b^{\gamma-1} \int_{t_{1}}^{t} e_{-p / a}(t, \sigma(s)) \eta(s) q(s) \theta^{\gamma-1}(s) \Delta s \\
& <-L b^{\gamma-1} \int_{t_{1}}^{t} e_{-p / a}(t, \sigma(s)) \eta(s) q(s) \theta^{\gamma-1}(s) \Delta s .
\end{aligned}
$$

Therefore, for all $l \in\left[t_{1}, \infty\right)_{\mathbb{T}}$,

$$
\begin{aligned}
& \int_{t_{1}}^{l} x^{\Delta}(u) \Delta u \\
& \quad<-L^{\frac{1}{\gamma-1}} b \int_{t_{1}}^{l}\left(\frac{1}{\eta(u) a(u)} \int_{t_{1}}^{u} e_{-p / a}(u, \sigma(s)) \eta(s) q(s) \theta^{\gamma-1}(s) \Delta s\right)^{\frac{1}{\gamma-1}} \Delta u .
\end{aligned}
$$

Letting $l \rightarrow \infty$ and using (3.27) in (3.32) yields $\lim _{l \rightarrow \infty} x(l)=-\infty$. This is a contradiction with the fact that $x(t)>0$. This completes the proof.

Remark 3.3 We note that (3.27) becomes (4.9) in [27] when $\eta(t) \equiv 1$, and so Theorem 3.5 in this paper includes Theorem 4.3 given in Zhang [27].

\section{Example}

In this section, we give an example to illustrate Theorem 3.1.

Example 4.1 Consider the second-order half-linear delay dynamic equation on time scales

$$
\begin{aligned}
& \left(\left|x^{\Delta}(t)\right|^{\gamma-2} x^{\Delta}(t)\right)^{\Delta}+\frac{1}{t \sigma(t)}\left|x^{\Delta}(t)\right|^{\gamma-2} x^{\Delta}(t)+\beta\left(\frac{\sigma(t)}{t}\right)^{\gamma-1}\left|x\left(\frac{t}{2}\right)\right|^{\gamma-2} x\left(\frac{t}{2}\right) \\
& =0, \quad t \geq 2,
\end{aligned}
$$

where $\gamma>1, \tau \circ \sigma=\sigma \circ \tau$.

Let

$$
\begin{array}{ll}
a(t)=1, & p(t)=\frac{1}{t \sigma(t)}, \quad q(t)=\beta\left(\frac{\sigma(t)}{t}\right)^{\gamma-1}, \\
f(u)=u, & \tau(t)=\frac{t}{2}, \quad \mu(t)<t .
\end{array}
$$

It is easy to see that the condition $\left(C_{2}\right)$ is satisfied, $\left(C_{3}\right)$ holds with $L=1$, and $\left(C_{1}\right)$ is satisfied as

$$
1-\mu(t) \frac{p(t)}{a(t)}>1-t \frac{1}{t \sigma(t)}>0 \quad \text { for } t \geq 2
$$

For any $t \geq 2$, we have

$$
R(t)=a^{\frac{1}{\gamma-1}}(t) \int_{2}^{t} a^{-\frac{1}{\gamma-1}}(s) \Delta s=t-2
$$


and we can find $0<k<1$ such that

$$
\begin{aligned}
& \alpha(t)=R(t) /(R(t)+\mu(t))=(t-2) /(\sigma(t)-2) \geq k t / \sigma(t), \\
& \alpha(\tau(t)) \geq k t / \sigma(t) .
\end{aligned}
$$

Next, by Lemma 2 in [29], from $\left(C_{1}\right)$, we get

$$
e_{-p / a}(t, 2) \geq 1-\int_{2}^{t} \frac{p(s)}{a(s)} \Delta s=1-\int_{2}^{t} \frac{1}{t \sigma(t)}=1-\left(\frac{1}{2}-\frac{1}{t}\right)>\frac{1}{2}
$$

and

$$
\int_{2}^{t}\left[\frac{1}{a(s)} e_{-p / a}\left(s, t_{0}\right)\right]^{\frac{1}{\gamma-1}} \Delta s=\int_{2}^{t}\left(e_{-p / a}\left(s, t_{0}\right)\right)^{\frac{1}{\gamma-1}} \Delta s>\left(\frac{1}{2}\right)^{\frac{1}{\gamma-1}}(t-2) .
$$

Letting $t \rightarrow \infty$ in the above inequality, we obtain that (1.5) holds. Take $\delta(t)=t$ for all $t \geq 2$. Then

$$
\begin{aligned}
& \limsup _{t \rightarrow \infty} \int_{2}^{t}\left(L q(s)(\alpha(\tau(s)))^{\gamma-1}-\frac{a(\tau(s))\left|\frac{\delta^{\Delta}(s)}{\delta(s)}-\frac{p(s)}{a(s)}\right|^{\gamma}}{\gamma^{\gamma}\left(\tau^{\Delta}(s)\right)^{\gamma-1}}\right) \delta(s) \Delta s \\
& \geq \limsup _{t \rightarrow \infty} \int_{2}^{t}\left(\beta\left(\frac{\sigma(s)}{s}\right)^{\gamma-1}\left(\frac{k s}{\sigma(s)}\right)^{\gamma-1}-\frac{\mid \frac{1}{s}-\frac{1}{s \sigma(s)}}{\gamma^{\gamma}\left(\frac{1}{2}\right)^{\gamma-1}}\right) s \Delta s \\
& >\limsup \int_{t \rightarrow \infty}^{t}\left(\beta k^{\gamma-1}-\frac{2^{\gamma-1}}{\gamma^{\gamma} s^{\gamma-1}}\right) \Delta s \\
& \geq \limsup _{t \rightarrow \infty} \int_{2}^{t}\left(\beta k^{\gamma-1}-\frac{1}{\gamma^{\gamma}}\right) \Delta s=\infty,
\end{aligned}
$$

if $\beta>1 / \gamma^{\gamma} k^{\gamma-1}$. Then (4.1) is oscillatory by Theorem 3.1 when $\beta>1 / \gamma^{\gamma} k^{\gamma-1}$.

\section{Competing interests}

The authors declare that they have no competing interests.

\section{Authors' contributions}

The authors declare that the study was realized in collaboration with the same responsibility. All authors read and approved the final manuscript.

\section{Acknowledgements}

This research is supported by the Natural Science Foundation of China (11071143), Natural Science Outstanding Youth Foundation of Shandong Province (JQ201119) and supported by Shandong Provincial Natural Science Foundation (ZR2012AM009), also supported by Natural Science Foundation of Educational Department of Shandong Province (J11LA01)

Received: 13 March 2013 Accepted: 8 May 2013 Published: 23 May 2013

\section{References}

1. Hilger, S: Analysis on measure chains - a unified approach to continuous and discrete calculus. Results Math. 18, 18-56 (1990)

2. Bohner, M, Peterson, A: Dynamic Equations on Time Scales: An Introduction with Applications. Birkhäuser, Boston (2001)

3. Bohner, M, Peterson, A: Advances in Dynamic Equations on Time Scales. Birkhäuser, Boston (2003)

4. Erbe, L: Oscillation criteria for second order linear equations on a time scale. Can. Appl. Math. Q. 9, 345-375 (2001)

5. Erbe, L, Peterson, A, Rehák, P: Comparison theorems for linear dynamic equations on time scales. J. Math. Anal. Appl. 275, 418-438 (2002) 
6. Grace, SR, Agarwal, RP, Kaymakcalan, B, Sae-Jie, W: Oscillation theorems for second order nonlinear dynamic equations. J. Appl. Math. Comput. 32, 205-218 (2010)

7. Hassan, TS: Oscillation criteria for half-linear dynamic equations on time scales. J. Math. Anal. Appl. 345, 176-185 (2008)

8. Sahiner, Y: Oscillation of second-order delay differential equations on time scales. Nonlinear Anal. TMA 63, 1073-1080 (2005)

9. Sun, S, Han, Z, Zhang, C: Oscillation of second-order delay dynamic equations on time scales. J. Appl. Math. Comput. 30, 459-468 (2009)

10. Sun, S, Han, Z, Zhao, P, Zhang, C: Oscillation for a class of second order Emden-Fowler delay dynamic equations on time scales. Adv. Differ. Equ. 2010, Article ID 642356 (2010)

11. Han, Z, Sun, S, Li, T, Zhang, C: Oscillatory behavior of quasilinear neutral delay dynamic equations on time scales. Adv. Differ. Equ. 2010, Article ID 450264 (2010)

12. Han, Z, Sun, S, Shi, B: Oscillation criteria for a class of second order Emden-Fowler delay dynamic equations on time scales. J. Math. Anal. Appl. 334, 847-858 (2007)

13. Han, Z, Li, T, Sun, S, Zhang, C: Oscillation for second-order nonlinear delay dynamic equations on time scales. Adv. Differ. Equ. 2009, Article ID 756171 (2009)

14. Sun, Y, Han, Z, Li, T, Zhang, G: Oscillation criteria for second order quasi-linear neutral delay dynamic equations on time scales. Adv. Differ. Equ. 2010, Article ID 512437 (2010)

15. Li, T, Han, Z, Sun, S, Zhao, Y: Oscillation results for third order nonlinear delay dynamic equations on time scales. Bull. Malays. Math. Soc. 34(3), 639-648 (2011)

16. Han, Z, Li, T, Sun, S, Cao, F: Oscillation criteria for third order nonlinear delay dynamic equations on time scales. Ann Pol. Math. 99, 143-156 (2010)

17. Han, Z, Li, T, Sun, S, Zhang, C: Oscillation behavior of third order neutral Emden-Fowler delay dynamic equations on time scales. Adv. Differ. Equ. 2010, Article ID 586312 (2010)

18. Sun, Y, Han, Z, Sun, Y, Pan, Y: Oscillation theorems for certain third order nonlinear delay dynamic equations on time scales. Electron. J. Qual. Theory Differ. Equ. 2011, 75 (2011)

19. Zhang, S, Wang, Q: Oscillation of second-order nonlinear neutral dynamic equations on time scales. Appl. Math. Comput. 216, 2837-2848 (2010)

20. Zhao, A, Wang, Y, Yan, J: Oscillation criteria for second-order nonlinear differential equations with nonlinear damping Comput. Math. Appl. 56, 542-555 (2008)

21. Hassan, TS, Erbe, L, Peterson, A: Oscillation of second order superlinear dynamic equations with damping on time scales. Comput. Math. Appl. 59, 550-558 (2010)

22. Erbe, L, Hassan, TS, Peterson, A: Oscillation criteria for nonlinear damped dynamic equations on time scales. Appl. Math. Comput. 203, 343-357 (2008)

23. Saker, SH, Agarwal, RP, O'Regan, D: Oscillation of second-order damped dynamic equations on time scales. J. Math. Anal. Appl. 330, 1317-1337 (2007)

24. Bohner, M, Erbe, L, Peterson, A: Oscillation for nonlinear second order dynamic equations on a time scale. J. Math. Anal. Appl. 301, 491-507 (2005)

25. Zafer, A: On oscillation and nonoscillation of second order dynamic equations. Appl. Math. Lett. 22, 136-141 (2009)

26. Chen, W, Han, Z, Sun, S, Li, T: Oscillation behavior of a class of second-order dynamic equations with damping on time scales. Discrete Dyn. Nat. Soc. 2010, 1-15 (2010)

27. Zhang, Q: Oscillation of second-order half-linear delay dynamic equations with damping on time scales. J. Comput. Appl. Math. 235, 1180-1188 (2011)

28. Hardy, GH, Littlewood, JE, Pólya, G: Inequalities, 2nd edn. Cambridge University Press, Cambridge (1988)

29. Bohner, M: Some oscillation criteria for first order delay dynamic equations. Far East J. Appl. Math. 18, 289-304 (2005)

doi:10.1186/1687-1847-2013-145

Cite this article as: Han et al.: Oscillation criteria for $p$-Laplacian delay dynamic equations with damping on time scales. Advances in Difference Equations 2013 2013:145.

\section{Submit your manuscript to a SpringerOpen ${ }^{\circ}$ journal and benefit from:}

- Convenient online submission

Rigorous peer review

- Immediate publication on acceptance

- Open access: articles freely available online

- High visibility within the field

- Retaining the copyright to your article

Submit your next manuscript at $>$ springeropen.com 